\title{
Overexpression of the $h$ Biot 2 gene is associated with development of human cervical cancer
}

\author{
YANG-MEI SHEN ${ }^{1}$, XIANG HE$^{2}$, HONG-XIN DENG ${ }^{3}$, YU-PING XIE ${ }^{4}$, \\ CHUN-TING WANG ${ }^{3}$, YU-QUAN WEI ${ }^{3}$ and XIA ZHAO ${ }^{2}$
}

\author{
Departments of ${ }^{1}$ Pathology, ${ }^{2}$ Gynecology and Obstetrics, ${ }^{3}$ State Key Laboratory of Biotherapy of Human Diseases, \\ ${ }^{4}$ Regenerative Medicine Research Center, West China Hospital of Sichuan University, Chengdu, P.R. China
}

Received July 26, 2010; Accepted August 25, 2010

DOI: $10.3892 /$ or_00001044

\begin{abstract}
The novel gene human Biot2 ( $h$ Biot2) was first reported by our laboratory. Previously, we indicated its function of proliferation and carcinogenesis in human endometrial cancer. In the present study, we aimed to investigate whether $h$ Biot 2 played a similar role in human cervical cancer. We tested $h$ Biot 2 expression profile in cervical cancer, the corresponding adjacent normal tissues, normal cervix and the cervical cancer cell lines by RT-PCR and compared the mean value of $h$ Biot 2 expression between cervical cancer and normal cervix, and cervical cancer with or without lymphatic metastasis by real-time PCR. The location of $h$ Biot 2 in normal cervix and cancer tissues together with the corresponding adjacent normal tissues was determined by RNA-RNA in situ hybridization (ISH). $h$ Biot 2 expression in the cervical cancer $(20 / 25)$, the corresponding adjacent normal tissues (3/12), normal cervix (17/18) and the cervical cell lines (2/3) was shown by RT-PCR. The mean value of $h$ Biot 2 expression was higher in the cervical cancer than in the normal cervix $(0.478 \pm 1.612$ vs. $0.091 \pm 0.107, \mathrm{P}=0.0004)$, higher in the lymphatic metastasis than in the non-lymphatic metastasis in the cervical cancer $(1.117 \pm 2.483 \mathrm{vs}$. $0.052 \pm 0.071, \mathrm{P}=0.014)$. $h$ Biot 2 expression location was mainly in the parenchymal cells of the cervical cancer and normal cervix rather than in the stromal cells. Overexpression of $h$ Biot 2 is associated with early and interim development of human cervical cancer.
\end{abstract}

\section{Introduction}

The Human Genome Project (HGP) was launched in 1990 and a draft of the sequences of the human genome was

Correspondence to: Dr Xia Zhao, Department of Gynecology, West China Second Hospital of Sichuan University, no. 20, Section 3, South People's Road, Chengdu, Sichuan 610041, P.R. China E-mail: xia-zhao@126.com

Key words: cervical cancer, $h \mathrm{Biot} 2$, RT-PCR, in situ hybridization, overexpression reported in February 2001. The euchromatic sequence of the human genome with high accuracy and nearly complete coverage was reported in 2004 (International Human Genome Sequencing Consortium, 2004). Following the publication of this project, much importance was focused on finding, cloning and analyzing novel genes to determine their sequences, as well as their expression locations and functions (1). As altered gene expression is a common feature of neoplastic cells, researchers had sought to identify genes which are abundantly or specifically expressed in tumor tissues when contrasted with corresponding adjacent normal tissue $(2,3)$. This comparison is potentially a very useful tool in the understanding of carcinogenesis and tumor progression.

Rat Biot 2 is a novel gene first reported and named by our laboratory (GenBank accession no. AY845219). A portion of this novel gene was identified using the rabbit serum immunized with human mammary cancer and ovarian cancer cells to screen the rat testis cDNA expression library using the serological analysis of the recombinant cDNA expression library (SEREX) approach (4). The full length cDNA of Rat Biot2 was next obtained by the Rapid Amplification of cDNA Ends (RACE) technique and the Expressed Sequence Tags (EST) technique. The previous study and the bioinformatics analysis revealed that Rat Biot2 may correlate with sperm development in rat and have potential function to stimulate proliferation of cells $(5,6)$. $h$ Biot2 (human Biot2), as the human homologous gene of Rat Biot2, was obtained using homology analysis in NCBI (National Center for Biotechnology Information) (GenBank accession no. AK057324). The fulllength cDNA sequence of $h$ Biot2 is located in chromosome 10 (10p11.22) with $1819 \mathrm{bp}$ and composed of 18 exons and 17 introns. The open reading frame (ORF) is $804 \mathrm{bp}$ and encodes the protein of 267 amino acids. The ORF is composed of 9 exons and 8 introns. In a previous study, it was indicated that $h$ Biot 2 played an important role in carcinogenesis in human endometrial cancer (7). Invasive cervical cancer is a leading cause of cancer death in women, however, clinical outcomes vary significantly and are difficult to predict (8). Therefore, it is necessary and urgent to search for an effective tumor marker to predict and diagnose cervical cancer in its early stage. In the current study, we studied the expression profile of $h \mathrm{Biot} 2$ in cervical cancer and the relationships between expression value and clinicopathological variables. 


\section{Materials and methods}

Tissues. For scientific purposes, all samples were collected with the permission of the ethics committee of Second West China Hospital of Sichuan University and with the consent of the patients or the relatives. All tissues were from patients who underwent an operation between September 2005 to May 2006 in the Department of Gynecology and Obstetrics (The Second West China Hospital of Sichuan University, Chengdu, Sichuan, China), which included normal cervix $(n=18)$, cervical cancer $(n=25)$ and the corresponding adjacent normal tissues $(n=12)$. The corresponding adjacent normal tissues were obtained $5 \mathrm{~cm}$ beyond the boundary of the cancerous tissue, which were myometrium in essence. Each samples were cut into two pieces, one was harvested and embedded in Tissue-Tek OCT compound (Sakura, Tokyo, Japan) within $10 \mathrm{~min}$ after resection from the patients and snap-frozen in liquid nitrogen before storage at $-80^{\circ} \mathrm{C}(9)$. The other piece was fixed in fresh $10 \%$ neutral-buffered formalin for $24 \mathrm{~h}$ at room temperature.

Cell lines. The cervical cancer cell line HeLa was obtained from the American Type Culture Collection (ATCC, Manassas, USA). Another two cervical carcinoma cell lines Siha, Caski were kindly provided by Gynecological Cancer Laboratory, Second West China Hospital of Sichuan University (The Gynecological Cancer Laboratory obtained the cell lines from ATCC). Cell lines were cultured in DMEM and RPMI-1640 medium supplemented with $10 \%$ fetal bovine serum and antibiotics (100 U/ml penicillin and $100 \mu \mathrm{g} / \mathrm{ml}$ streptomycin) (10-14). Cells were incubated at $37^{\circ} \mathrm{C}$ in humidified atmosphere of $5 \% \mathrm{CO}_{2}$. Cells $\left(1 \times 10^{7}\right)$ were collected and washed with PBS twice before they were treated with $1 \mathrm{ml}$ of TRIzol reagent for RNA isolation.

Reverse transcription PCR (RT-PCR). Total RNA from fresh, rapidly frozen tissues and cultured cells samples were isolated with single-step isolation method using TRIzol reagent (15) (Invitrogen, Tokyo, Japan) according to the manufacturer's instructions. Total RNA were used at once or stored in $-80^{\circ} \mathrm{C}$ until used.

The primers for $h$ Biot 2 were designed with the Jellyfish Software. The primer sequences are shown in Table I. RTPCR was performed with the One-step Reverse transcription PCR reagent (Takara) according to the manufacturer's instructions. The glyceraldehydes-3-phosphate dehydrogenase (GAPDH) gene was used as an internal control. Reverse transcription was carried at $42^{\circ} \mathrm{C}$ for 50 min using $1 \mu 1$ RNA in $25 \mu 1$ reaction system. Amplification reactions were 40 cycles $\left(94^{\circ} \mathrm{C} 30 \mathrm{sec}, 55^{\circ} \mathrm{C} 30 \mathrm{sec}, 72^{\circ} \mathrm{C} 30 \mathrm{sec}\right)$. The RT-PCR products were separated on a $1 \%$ agarose (GeneTech, Co. Limited) gel and visualized with ethidium bromide.

Quantitative real-time reverse transcription-PCR. Quantitative RT-PCR analysis was performed using an ABI PRISM 7000 sequence detection system (Applied Biosystems). Total RNA was completely the same as the RNA used in RT-PCR. Primers and TaqMan-probe for $h$ Biot 2 were designed with the assistance of the Invitrogen Co. The primers and probe sequences are listed in Table I. 
Real-time PCR were performed in two steps according to the manufacturer's instructions (code nos.: DRR035S and DRR039S, Takara). The first step was reverse transcription using $2 \mu \mathrm{l}$ RNA in $20 \mu 1$ reaction system. Samples were incubated for $15 \mathrm{~min}$ at $42^{\circ} \mathrm{C}$ and for $2 \mathrm{~min}$ at $95^{\circ} \mathrm{C}$. The second step was amplification reactions. The reaction conditions were as follows: $95^{\circ} \mathrm{C}$ for $2 \mathrm{~min}$, followed by $95^{\circ} \mathrm{C}$ $(10 \mathrm{sec}), 50^{\circ} \mathrm{C}(10 \mathrm{sec})$ and $72^{\circ} \mathrm{C}(34 \mathrm{sec})$ for 45 cycles $(16-18)$. To obtain the relative quantitation of gene expression, a validation experiment was done to test that the target ( $h$ Biot2) and reference (GAPDH) efficiencies of amplification were equal at different template dilutions (19). Target and reference probe sets were amplified in triplicates in separate wells of 96-well plates and data were averaged from the values obtained in each reaction $(20,21)$. Results were analyzed using ABI PRISM sequence detection system software (version 2.1 Applied Biosystems), which was the calculation of $\Delta \mathrm{Ct}$ $\left(\Delta \mathrm{Ct}=\right.$ average $\mathrm{Ct}_{h \mathrm{Biot} 2}$ - average $\left.\mathrm{Ct}_{\mathrm{GAPDH}}\right)$. The expression quantities of $h$ Biot 2 was normalized to that of GAPDH with a ratio using $2^{-\Delta C t}$. The procedure used has been described elsewhere $(22,23)$. Real-time PCR was performed to analyze $h$ Biot2 expression in normal cervix and cervical cancer.

In situ hybridization (ISH). Before and during the processing of hybridization, all equipment and materials were treated with $0.1 \%$ DEPC. The frozen samples embedded in OCT compound were cut in $5 \mu \mathrm{m}$ thick sections and mounted on $\alpha$-aminopropyl triethoxysilane (APES)-coated slides. They were stored at $-80^{\circ} \mathrm{C}$ until needed (15).

The gene $h$ Biot 2 fragment was subcloned into the pGEM-T vector (Promega). The recombinant PGEM ${ }^{\circledR}-\mathrm{T}$ Easy Vector/ $h$ Biot 2 plasmids were isolated according to the instruction of Invitrogen PureLink ${ }^{\mathrm{TM}}$ Quick Plasmid Miniprep kit (Invitrogen Corp., Carlsbad, USA) and then enzymatic cut by BamHI and $X o h \mathrm{I}$, respectively. The enzymatic cutting products were purified according to the instruction of EZ-10 Spin Column DNA Gel Extraction kit (Imperial Bio-Medic Lit Corp., Madhyamarg, India) for the temple DNA of the anti-sense RNA probe and sense RNA probe, respectively. Digoxigeninlabeled sense and anti-sense riboprobes were prepared by in vitro transcription using a DIG RNA Labeling kit (SP6/T7; Roche Diagnostics GmbH, Penzberg, Germany) according to the manufacturer's instructions $(20,24)$. The probes were stored at $-80^{\circ} \mathrm{C}$ and used within two months.

Prior to the procedure of ISH, the sections stored at $-80^{\circ} \mathrm{C}$ were recovered to room temperature and heated the sections in a stove for $2 \mathrm{~h}$ at $60^{\circ} \mathrm{C}$ to fix the RNA in the tissues. The process of ISH was performed under the direction of protocols $(15,25,26)$. The negative controls were determined by the substitution of sense for the anti-sense probe. The cells stained blue were identified as positive cells that could express $h$ Biot 2 .

Histological analysis. The other piece of the tissue samples fixed in fresh $10 \%$ neutral-buffered formalin were cut into $5 \mu \mathrm{m}$ sections and stained with hematoxylin and eosin (H\&E). These sections were examined by experienced pathologists under a light microscopy to confirm histology.

Statistical analysis. Signed test was used to analyze the difference between the cervical cancer and the normal cervix,
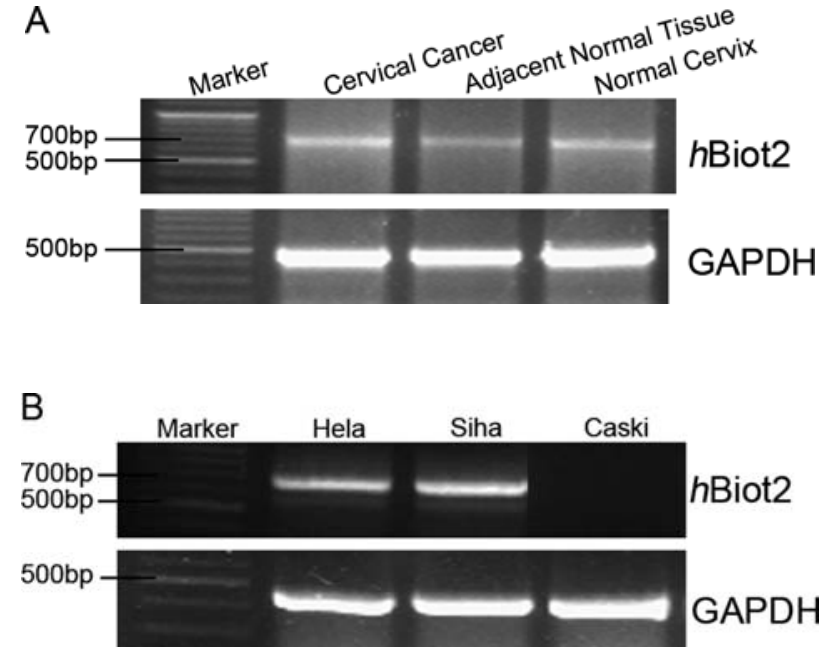

Figure 1. Using RT-PCR to detect the $h$ Biot2 (700 bp) expression in cervical cancer tissues and cell lines. (A) $h$ Biot 2 was detected in cervical cancer (20/25), the corresponding adjacent normal tissues (3/12) and normal cervix (17/18). (B) $h$ Biot 2 was detected in the cervical cancer cell lines Hela, and Siha, but not in Caski.

Man-Whitney U test was used to analyze the cervical cancer patients with or without lymphatic metastasis. Statistical analysis was performed using the SPSS 16.0 statistical software (SPSS Inc., Chicago, USA) and statistical significance was set at $\mathrm{P}<0.05$.

\section{Results}

hBiot2 expression in normal cervix, cervical cancer and the corresponding adjacent normal tissues. Twenty-five cases of cervical cancer together with 12 cases of the corresponding adjacent normal tissues, as well as 18 cases of normal cervix were involved in this study. The cervical cancers were squamous cell cancer and the FIGO (International Federation of Gynecological and Obstetrics) stage was Ia-IIIc. The expression of $h$ Biot $2(700 \mathrm{bp}$ ) was detected in cervical cancer (20/25), the corresponding adjacent normal tissues (3/12) and normal cervix (17/18) by RT-PCR (Fig. 1A).

hBiot 2 expression in cervical cancer cell lines. The expression of $h$ Biot 2 was detected in the cervical cancer cell lines Hela, and Siha, but not in Caski by RT-PCR (Fig. 1B).

The mean expression of hBiot 2 both in the cervical cancer and the normal cervix in real-time $R T-P C R$. Since the expression frequency of $h$ Biot 2 in cervical cancer (20/25) and in normal cervix (17/18) was similar, we further performed real-time RT-PCR in order to investigate expression value differences of $h \operatorname{Biot} 2$ between them. The mean value of $h$ Biot 2 expression was higher in the cervical cancer than in the normal cervix $(0.478 \pm 1.612$ vs. $0.091 \pm 0.107$, $\mathrm{P}=0.0004$ ) (Fig. 2A).

In cervical cancer, 2 groups were separated based on with or without lymphatic metastasis. Higher expression was seen in lymphatic metastasis compared with non-lymphatic metastasis $(1.117 \pm 2.483$ vs. $0.052 \pm 0.071, \mathrm{P}=0.014$ ) (Fig. $2 \mathrm{~B}$ ). 
A

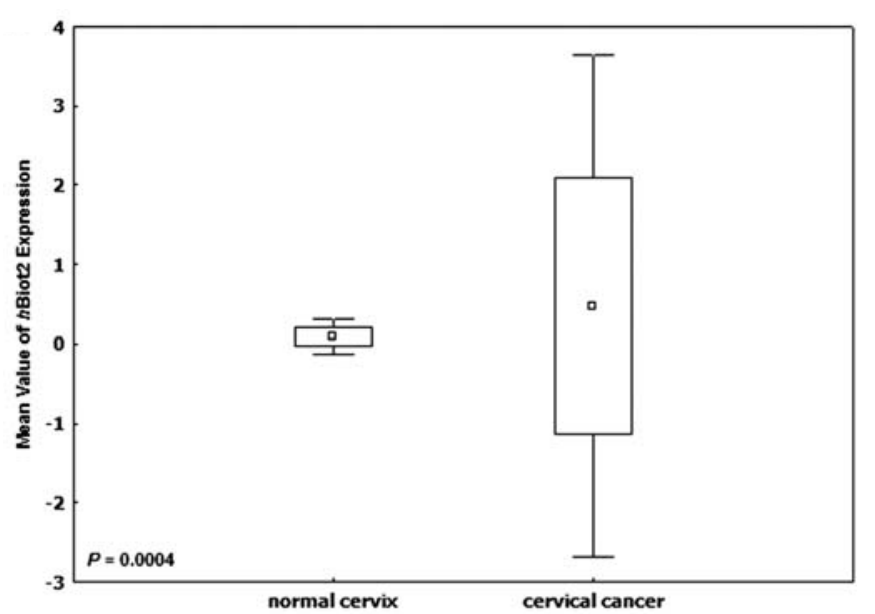

B

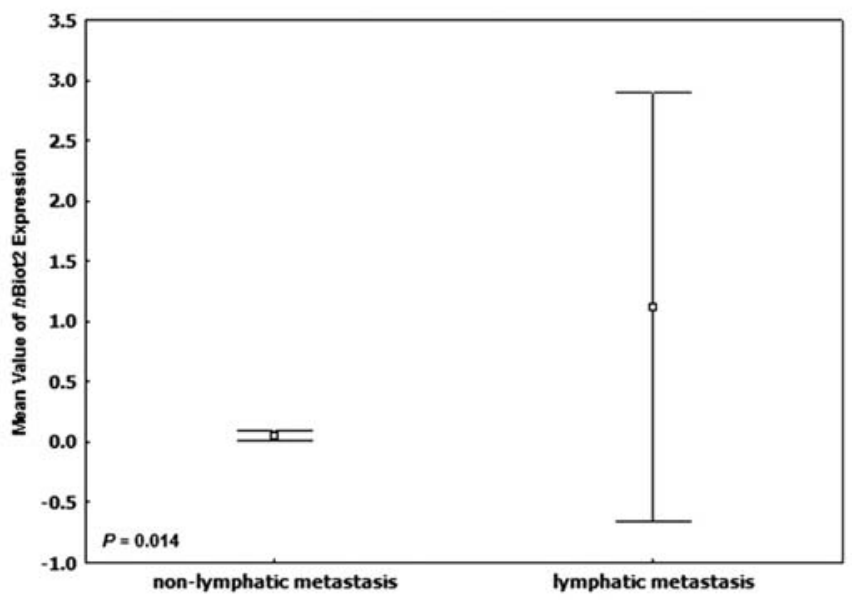

Figure 2. Using real-time PCR to detect the mean value of $h$ Biot2 expression in cervical cancer and normal cervix. (A) The mean value of $h$ Biot2 expression was higher in the cervical cancer than in normal cervix ( $0.478 \pm 1.612$ vs. $0.091 \pm 0.107, \mathrm{P}=0.0004)$. (B) Higher expression in lymphatic metastasis compared with non-lymphatic metastasis in cervical cancer $(1.117 \pm 2.483$ vs. $0.052 \pm 0.071, \mathrm{P}=0.014)$.

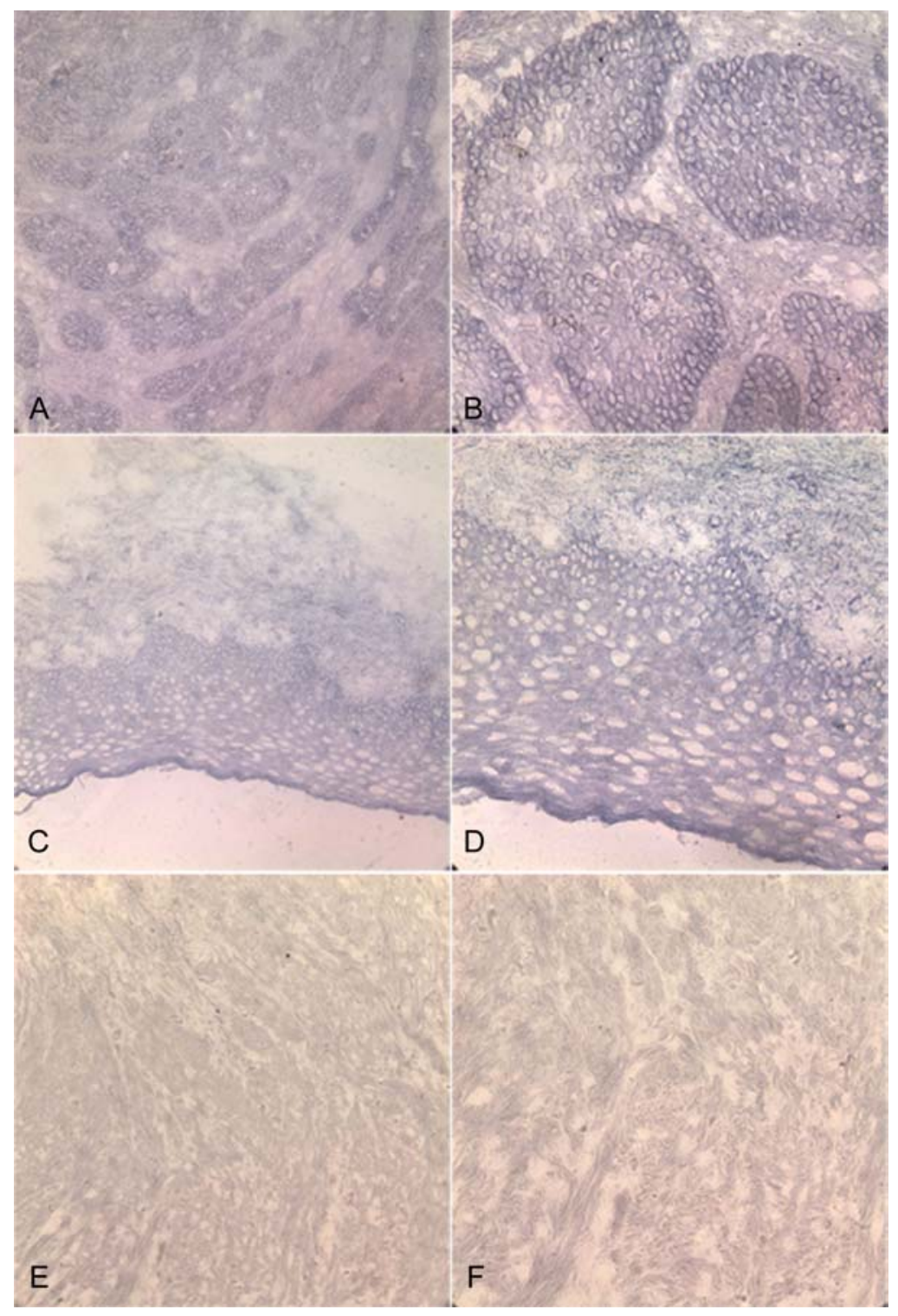

Figure 3. RNA expression of $h$ Biot2 was determined by ISH on the frozen sections in cervical cancer, the corresponding adjacent normal tissues and normal cervix (x200 in the left, $\mathrm{x} 400$ in the right). $h$ Biot 2 expressed strongly in the cytoplasm of tumor cells (A and B) and normal cervix (C and D). Corresponding adjacent normal tissues (myometrium in essence) did not express $h$ Biot2 (E and F). 
RNA expression of hBiot 2 determined by ISH in cervical cancer, the corresponding adjacent normal tissues and normal cervix. In order to reveal what type of cells expressing $h$ Biot 2 , we performed ISH to localize $h \mathrm{Biot} 2$ expression in mRNA level. The cells expressing $h$ Biot 2 were identified by the blue stain in the cytoplasma. hBiot 2 expression was mainly detected in the cervical cancer and normal cervix cells, which were detected only in the squamous cells in the malignancy of cervix and normal cervix, respectively. $h$ Biot 2 was only slightly expressed in normal myometrium (Fig. 3).

\section{Discussion}

One of the most important characteristics of cancer cells is the increased ability to proliferate and the decreased susceptibility to apoptosis (27). Moreover, studying the expression profile of a novel gene in tissues could help to determine the function of the gene and better understand the mechanism of carcinogenesis and cancer progression (28-30). In a previous study, we identified that $r$ Biot 2 gene could stimulate proliferation of cells in rat and mouse $(5,6)$, and $h$ Biot 2 might play an important role in the development of human endometrial cancer (7). Expression of $h$ Biot 2 was higher in endometrial cancer than in the corresponding normal endometrium, and the expression was higher in poorly differentiated tumors compared with well differentiated ones. The results indicated that $h$ Biot 2 may have a potential function in carcinogenesis. In the current study, we investigated the expression of $h \mathrm{Biot} 2$ in human cervical cancer and cervical cancer cell lines, as the first study investigating the novel gene $h$ Biot 2 expression in human cervical cancer.

In the present study, it was shown that $h \operatorname{Biot} 2$ was expressed in cervical cancer, the corresponding adjacent normal tissues and normal cervix by RT-PCR. The expression frequency was quite different between in the cervical cancer, normal cervix and the corresponding adjacent normal tissues, which was lower in the corresponding adjacent normal tissues compared with cervical cancer and normal cervix, but the expression frequency was similar between the cervical cancer and normal cervix. As mentioned above, the corresponding adjacent normal tissues were myometrium in essence. Therefore, it seemed the difference among them was caused by epithelia cells instead of stromal cells, such as myometrium. We further investigated the expression amount of $h$ Biot 2 both in cervical cancer and normal cervix using real-time PCR. The results showed that mean value of $h$ Biot 2 expression was higher in cervical cancer than normal cervix, higher in lymphatic metastasis than non-lymphatic metastasis in cervical cancer. Thus, $h$ Biot 2 expression increased from normal cervix to cancer and also related to the metastasis potential, that is the higher the expression, the higher the metastasis potential. Taken together, $h$ Biot 2 may play an important role in the development of cervical cancer.

Moreover, we studied the cervical cancer cell lines, including Hela, Siha and Caski using RT-PCR. It was shown that $h$ Biot 2 was expressed both in Hela and Siha, no expression was seen in Caski. We speculated this could be because the Caski cell line was taken and originated from metastasis in the small intestine rather than the primary site, while the Hela and Siha cell lines were taken and originated from the cervix. The bioinformatics of tumor cells might change greatly once distant metastasis occur and expressed none or less $h$ Biot 2 . However, combined with the results mentioned above, the trend was for higher expression of $h$ Biot2, the the higher metastasis potential. We further suggest that $h$ Biot 2 was only related to local metastasis instead of distant metastasis, in other words, $h$ Biot 2 focused on the early and interim stage in the development of cervical cancer and played a lesser role in the late development. These results were consistent with other unpublished results, which found increasing $h$ Biot 2 expression during the early and interim development of colorectal cancer (CRC), and then decreased in the late period of $\mathrm{CRC}$, while almost nonexistation in liver metastasis. Therefore, overexpression of $h$ Biot 2 was associated with early and interim development of cervical cancer.

To rule out that the $h$ Biot 2 difference of expression was caused by the stromal cells, being a possible confounding variable in this study, we determined the localization of $h$ Biot 2 gene expressed in the cervical cancer, the corresponding adjacent normal tissues and normal cervix. The expression locations of $h$ Biot 2 on the frozen sections in cervical cancer, the corresponding adjacent normal tissues and normal cervix were performed by ISH of RNA level. Our results showed that $h \mathrm{Biot} 2$ expressed in the normal epithelial and tumor cells of the cervical cancer but not in the stromal cells and myometrium, which indicated that the value of $h$ Biot 2 , determined by real-time PCR, in the both normal cervix and cervical cancer samples represented normal epithelial cells and cancer cells. This result agreed with the result that both cervical cancer and normal cervix expressed $h$ Biot2, while the corresponding adjacent normal tissues (myometrium in essence) were only slightly expressed.

In conclusion, $h$ Biot 2 was expressed in epithelium of normal cervix and cervical cancer. Its overexpression was associated with early and interim development of cervical cancer and possibly could be a diagnostic factor in early stage of cervical cancer.

\section{References}

1. Wiemann S, Weil B, Wellenreuther R, et al: Toward a catalog of human genes and proteins: sequencing and analysis of 500 novel complete protein coding human cDNAs. Genome Res 11: $422-435,2001$

2. Mathioudaki K, Scorilas A, Papadokostopoulou A, et al: Expression analysis of BCL2L12, a new member of apoptosisrelated genes, in colon cancer. Biol Chem 385: 779-783, 2004.

3. Song J, Yang W, Shih IeM, Zhang Z and Bai J: Identification of BCOX1, a novel gene overexpressed in breast cancer. Biochim Biophys Acta 1760: 62-69, 2006.

4. Chen YT, Scanlan MJ, Sahin U, et al: A testicular antigen aberrantly expressed in human cancers detected by autologous antibody screening. Proc Natl Acad Sci USA 94: 1914-1918, 1997.

5. Hanshuo Y, Chunting W, Rui W, et al: Characterization of a novel rat gene RTAP2a, screened by cross-reactive SEREX, restrictedly expressed in testis. J Biosci Bioeng 107: 589-595, 2009.

6. Wang CT, Zhang P, Wang YS, et al: RNA interference against Biot2, a novel mouse testis-specific gene, inhibits the growth of tumor cells. Cell Mol Biol Lett 14: 363-376, 2009.

7. Yangmei S, Xiang H, Hongxin D, et al: Expression of human Biot 2 and its potential function on carcinogenesis in endometrial cancer. Acta Obstet Gynecol Scand 86: 1503-1509, 2007. 
8. Hu X, Schwarz JK, Lewis JS Jr, et al: A microRNA expression signature for cervical cancer prognosis. Cancer Res 70: 1441-1448, 2010.

9. Iacobuzio-Donahue CA, Maitra A, Shen-Ong GL, et al: Discovery of novel tumor markers of pancreatic cancer using global gene expression technology. Am J Pathol 160: 1239-1249, 2002.

10. Kloth JN, Fleuren GJ, Oosting J, De Menezes RX, Eilers PH, Kenter GG and Gorter A: Substantial changes in gene expression of Wnt, MAPK and TNF-alpha pathways induced by TGFbeta1 in cervical cancer cell lines. Carcinogenesis 26: 1493-1502, 2005.

11. Hiroi T, Someya A, Thompson W, Moss J and Vaughan M: GEP100/BRAG2: activator of ADP-ribosylation factor 6 for regulation of cell adhesion and actin cytoskeleton via E-cadherin and alpha-catenin. Proc Natl Acad Sci USA 103: 10672-10677, 2006.

12. Acconcia F, Barnes CJ and Kumar R: Estrogen and tamoxifen induce cytoskeletal remodeling and migration in endometrial cancer cells. Endocrinology 147: 1203-1212, 2006.

13. Meinhold-Heerlein I, Stenner-Liewen F, Liewen H, et al: Expression and potential role of Fas-associated phosphatase-1 in ovarian cancer. Am J Pathol 158: 1335-1344, 2001.

14. Jimeno A, Kulesza P, Kincaid E, et al: C-fos assessment as a marker of anti-epidermal growth factor receptor effect. Cancer Res 66: 2385-2390, 2006.

15. Nakamura Y, Nawata M and Wakitani S: Expression profiles and functional analyses of Wnt-related genes in human joint disorders. Am J Pathol 167: 97-105, 2005.

16. Livak KJ, Flood SJ, Marmaro J, Giusti W and Deetz K: Oligonucleotides with fluorescent dyes at opposite ends provide a quenched probe system useful for detecting PCR product and nucleic acid hybridization. PCR Methods Appl 4: 357-362, 1995.

17. Heid CA, Stevens J, Livak KJ and Williams PM: Real time quantitative PCR. Genome Res 6: 986-994, 1996.

18. Gibson UE, Heid CA and Williams PM: A novel method for real time quantitative RT-PCR. Genome Res 6: 995-1001, 1996.

19. Ma X, Chen K, Huang S, et al: Frequent activation of the hedgehog pathway in advanced gastric adenocarcinomas. Carcinogenesis 26: 1698-1705, 2005.
20. Tanaka Y, Miyamoto S, Suzuki SO, et al: Clinical significance of heparin-binding epidermal growth factor-like growth factor and a disintegrin and metalloprotease 17 expression in human ovarian cancer. Clin Cancer Res 11: 4783-4792, 2005.

21. Modena P, Lualdi E, Facchinetti F, Galli L, Teixeira MR, Pilotti S and Sozzi G: SMARCB1/INI1 tumor suppressor gene is frequently inactivated in epithelioid sarcomas. Cancer Res 65: 4012-4019, 2005.

22. Bustin SA: Absolute quantification of mRNA using real-time reverse transcription polymerase chain reaction assays. J Mol Endocrinol 25: 169-193, 2000.

23. Bowden NA, Weidenhofer J, Scott RJ, Schall U, Todd J, Michie PT and Tooney PA: Preliminary investigation of gene expression profiles in peripheral blood lymphocytes in schizophrenia. Schizophr Res 82: 175-183, 2006.

24. Irla M, Puthier D, Le Goffic R, et al: Spatial, a new nuclear factor tightly regulated during mouse spermatogenesis. Gene Expr Patterns 3: 135-138, 2003.

25. Iacobuzio-Donahue CA, Ryu B, Hruban RH and Kern SE: Exploring the host desmoplastic response to pancreatic carcinoma: gene expression of stromal and neoplastic cells at the site of primary invasion. Am J Pathol 160: 91-99, 2002.

26. Suzuki SO and Iwaki T: Non-isotopic in situ hybridization of CD44 transcript in formalin-fixed paraffin-embedded sections. Brain Res Brain Res Protoc 4: 29-35, 1999.

27. Green DR and Evan GI: A matter of life and death. Cancer Cell 1: 19-30, 2002.

28. Chen YC, Davidson B, Cheng CC, et al: Identification and characterization of membralin, a novel tumor-associated gene, in ovarian carcinoma. Biochim Biophys Acta 1730: 96-102, 2005.

29. Korkmaz CG, Korkmaz KS, Kurys P, et al: Molecular cloning and characterization of STAMP2, an androgen-regulated six transmembrane protein that is overexpressed in prostate cancer. Oncogene 24: 4934-4945, 2005.

30. Simon I, Zhuo S, Corral L, Diamandis EP, Sarno MJ, Wolfert RL and Kim NW: B7-h4 is a novel membrane-bound protein and a candidate serum and tissue biomarker for ovarian cancer. Cancer Res 66: 1570-1575, 2006. 